\title{
Germs and their Operational Calculus
}

by

\section{J. MIKUSINSKI (Warszawa)}

1. Definition and general properties of germs. We say that two functions $f$ and $g$, defined in an interval $0 \leqslant t<T$, are equivalent, and we write $f \sim g$, if there is an interval $0 \leqslant t<t_{0}\left(t_{0} \leqslant T\right)$ in which $f(t)=g(t)$ holds. The set $x=\{f\}$ of all functions $k$ which are equivalent to a given function $f$ will be called the germ of $f$. If we know the germ of a function, we know more than its value at $t=0$, but less than its behaviour in any right neighbourhood of this point. Evidently, $f \sim g$ implies $\{f\}=\{g\}$, and conversely.

For real valued $f$ and $g$, we write $\{f\} \leqslant\{g\}$, if there is an interval $0 \leqslant t<t_{0}$ in which $f(t) \leqslant g(t)$ holds. This definition of inequality of germs is consistent, i.e. does not depend on the choice of representants of germs. By a representant of a germ $\{f\}$ we understand the function $f$ or any equivalent function. Evidently, the relation of inequality between germs has the following properties:

$1^{\circ} x \leqslant x$.

$2^{\circ}$ If $x \leqslant y$ and $y \leqslant z$, then $x \leqslant z$.

$3^{\circ}$ If $x \leqslant y$ and $y \leqslant x$, then $x=y$.

Let $\mathscr{G}$ denote the set of all real germs, i.e. of the germs of real-valued functions. Then, for any pair $x, y$ from $\mathscr{G}$, there is in $\mathscr{G}$ a third germ $z$ such that $x \leqslant z$ and $y \leqslant z$. In other words, $\mathscr{G}$ is a partially ordered set.

We define the arithmetic operations on germs as follows:

$$
\begin{gathered}
\{f\}+\{g\}=\{f+g\} ; \\
\{f\}\{g\}=\{f g\} .
\end{gathered}
$$

It is immediate that these definitions are consistent and that $\mathscr{G}$ is a commutative ring. If we identify numbers $\gamma$ with the germs $\{\gamma\}$ (of constant functions whose values are $\gamma$ ), then $\mathscr{G}$ becomes a commutative algebra and can be considered as an extension of the algebra of real numbers.

Note that there are positive germs which are less than any positive number, e.g. the germ of the linear function $f(t)=t$. Such germs can 
be called positive infinitesimals. Similarly we can introduce negative infinitesimals. More generally, the germ of any function $f$ such that

$$
\lim _{t \rightarrow 0+} f(t)=0
$$

(except for the trivial case $f(t) \equiv 0$ ) can be considered as an infinitesimal; it is greater than any negative number and less than any positive number.

Note finally that the ring $\mathscr{G}$ has divisors of zero and therefore can not be completed to a field.

2. Convolution ring of germs. A germ is said to be continuous if it has, among its representants, a continuous function. The set of real continuous germs is a subring of $\mathscr{G}$, when adopting definitions (1) and (2). This subring has also divisors of zero.

However, we can also define the product, alternatively, as a convolution rather than by (2). Then we obtain a ring without divisors of zero. By the convolution of germs we understand

$$
\{f\} *\{g\}=\{f * g\}
$$

where

$$
(f * g)(t)=\int_{0}^{\ell} f(t-\tau) g(\tau) d \tau .
$$

It is easy to see that this definition is consistent and that, under addition (1) and multiplication (3), the set of continuous germs is a ring. The fact that this ring has no divisors of zero follows easily from Titchmarsh's theorem on convolution.

In the preceding definitions of this section, it is irrelevant whether the germs are real or complex. By a complex germ $\{f\}$ we understand the set of all functions $k$ which are equivalent to a given complex valued function $f$. Let $\mathscr{C}$ denote the ring of complex continuous germs with convolution as multiplication. Through the whole remaining part of this paper the product $x y$ of germs $x, y$ will be understood in the sense of convolution. The meaning of powers $x^{n}(x \in \mathscr{C}, n$ positive integer $)$ is obvious.

We shall say that a power series of $x \in \mathscr{C}$

$$
\alpha_{1} x+\alpha_{2} x^{2}+\ldots \quad\left(\alpha_{n} \text { complex numbers }\right)
$$

converges to an element $u \in \mathscr{C}$, if for $x=\{f\}$ the series

$$
a_{1} f+\alpha_{2} f^{2}+\ldots
$$

(where $f^{n}$ denotes the $n$-th convolution power of the function $f$ ) converges, in an interval $0 \leqslant t<t_{0}$, uniformly to a function $r$ such that $u=\{r\}$.
We shall show that this definition of convergence is consistent, i.e. that the limit $u$, if exists, does not depend on the choice of the representant $f$ of the germ $x$. In fact, let $x=\{f\}=\{g\}$. Then, by definition, we have $f \sim g$, i.e. there exists an interval $0 \leqslant t<t_{1}$ in which $f(t)=g(t)$ holds. If (6) converges to $r$ uniformly in $0 \leqslant t<t_{0}$, then it does so in the interval $0 \leqslant t<t_{2}$ with $t_{2}=\min \left(t_{0}, t_{1}\right)$. Subsequently, the series $a_{1} g+\alpha_{2} g^{2}+\ldots$ converges also to $r$ uniformly in $0 \leqslant t<t_{2}$. Thus we obtain the same limit $\{r\}$, no matter what is the representant of $x$.

Note that the definition of convergence of a power series, given above, is different from the usual way. Traditionally, one defines first a convergence of sequences and then the convergence of series as sequences of partial sums. Such a traditional way fails in our case. In fact, it is natural to require, for purposes of Analysis, that the concept of convergence of sequences should satisfy the following conditions:

(i) If $x_{n}=x$, then $x_{n} \rightarrow x$.

(ii) If $\lambda_{n} \rightarrow 0\left(\lambda_{n}\right.$ numbers), then $\lambda_{n} x \rightarrow 0$.

(iii) If $\left|x_{n}\right| \leqslant y_{n}$ and $y_{n} \rightarrow 0$, then $x_{n} \rightarrow 0$.

It is clear in the above relation, how a product $\lambda x_{n}(\lambda$ number, $x \in \mathscr{C})$ and the modulus $|x|(x \in \mathscr{C})$ should be understood. Namely,

$$
\begin{aligned}
& \lambda\{f\}=\{\lambda f\}, \\
& |\{f\}|=\{|f|\} .
\end{aligned}
$$

It is also easily seen that definitions (7) and (8) are consistent and that

$$
\begin{gathered}
\lambda(x x)=(\lambda x) x, \quad \lambda(x+y)=\lambda x+\lambda y, \quad(\lambda+x) x=\lambda x+x x, \\
|x+y| \leqslant|x|+|y|,
\end{gathered}
$$

where $\lambda, x$ are numbers and $x, y \in \mathscr{C}$.

Now, let $x_{n}=f_{n}$, where

$$
f_{n}(t)= \begin{cases}t & \text { for } \quad 0 \leqslant t<\frac{1}{n}, \\ \frac{1}{n} & \text { for } \quad \frac{1}{n} \leqslant t<\infty\end{cases}
$$

and let $x=\{f\}$, where $f(t)=1$ for $0 \leqslant t<\infty$. Then (7) and (ii) imply that

$$
y_{n}=\frac{1}{n} x \rightarrow 0
$$

and, moreover, we have $\left|x_{n}\right|=x_{n} \leqslant y_{n}$. Thus, in view of (iii) we would have $x_{n} \rightarrow 0$. But, evidently, $x_{n}=x_{1} \neq 0$ for every $n=1,2, \ldots$, thus (i) would imply $x_{n} \rightarrow x_{1} \neq 0$ and the limit of $x_{n}$ would not be unique. 
Thus we can introduce in $\mathscr{C}$ no general convergence of sequences such that the limits of convergent sequences are unique and that properties (i), (ii) and (iii) are preserved. In spite of this fact it is sensible to define a convergence of power series, as has been done above.

Power series of germs have ordinary properties: If

$$
\alpha_{1} x+\alpha_{2} x^{2}+\ldots=u,
$$

then, for every number $\lambda$,

$$
\lambda \alpha_{1} x+\lambda \alpha_{2} x^{2}+\ldots=\lambda u .
$$

If (9) holds and, moreover,

$$
\begin{gathered}
\beta_{1} x+\beta_{2} x^{2}+\ldots=v \\
\left(\alpha_{1}+\beta_{1}\right) x+\left(\alpha_{2}+\beta_{2}\right) x^{2}+\ldots=u+v, \\
\gamma_{2} x^{2}+\gamma_{3} x^{3}+\ldots=u v
\end{gathered}
$$

and

with $\gamma_{n}=\alpha_{1} \beta_{n-1}+\ldots+\alpha_{n-1} \beta_{1}$.

The proofs of (10) and (11) are immediate. In order to prove (12), we write $x=\{f\}$ and assume that $(6)$ converges to $r$ uniformly in $0 \leqslant t<t_{0}$ and

$$
\beta_{1} f+\beta_{2} f^{2}+\ldots
$$

converges to $s$ uniformly in $0 \leqslant t<t_{1}$. Then both series (6) and (13) converge uniformly in $0 \leqslant t<t_{2}$ with $t_{2}=\min \left(t_{0}, t_{1}\right)$, to $r$ and $s$ respectively. Then series (12) converges to $r s$ uniformly in $0 \leqslant t<t_{2}$. Consequently (12) holds.

3. Distribution germs and operator germs. The germs considered so far will be called, in the sequel, function germs, in order to distinguish them from other kinds of germs we are going to introduce now.

Replacing in the preceding definition of germs the word function by distribution we obtain entities which may be called distribution germs. We consider first distributions defined in an interval $0 \leqslant t<T$ and we say that two such distributions $f$ and $g$ are equivalent, if $f=g$ holds in some interval $0 \leqslant t<t_{0}$. The set of all distributions which are equivalent to a given distribution is a distribution germ. In this definition, there is a small inexactitude, because distributions are usually defined in open sets, and in our case we need distributions which are defined in an interval which is half closed. This objection is not very serious and can be removed by a proper definition of distributions in such an interval. Instead of doing that we shall introduce a more general concept of germs,
namely operator germs.
We shall first recall the definition of an operator in an interval $0 \leqslant t<T$. We consider the set $C_{T}$ of complex-valued continuous functions in $0 \leqslant t<T$. The set $C_{T}$ is a ring under ordinary addition and convolution (4) as multiplication. We extend $O_{T}$ to a quotient ring $M_{T}$ and call elements of $M_{T}$ operators in $0 \leqslant t<T$. In particular, $M_{\infty}$ is a field (see [3]) and, for finite $T, M_{T}$ is a ring having divisors of zero (see [4]).

Two operators $f$ and $g$ will be said equivalent, if $f=g$ holds in some interval $0 \leqslant t<t_{0}\left(t_{0} \leqslant T\right)$. The meaning of the last expression needs an explanation. The operators $f$ and $g$ can be represented in the form

$$
f=\frac{p_{1}}{q_{1}} \quad \text { and } \quad g=\frac{p_{2}}{q_{2}}
$$

where $p_{1}, q_{1}, p_{2}$ and $q_{2}$ belong to $O_{T}$ and, besides, $q_{1}, q_{2}$ are functions which do not vanish identically in any interval $0 \leqslant t<t_{1}$. We say that

if

$$
f=g \quad \text { in } \quad 0 \leqslant t<t_{0},
$$

$$
p_{1} q_{2}=p_{2} q_{1} \quad \text { in } \quad 0 \leqslant t<t_{0},
$$

where the products are understood as convolutions. The meaning of the last equality is fully determined, for both sides of this equality are continuous functions. It remains to show that the definition of (15) does not depend on the choice of representation (14). Let us take another representation

$$
f=\frac{p_{3}}{q_{3}} \quad \text { and } \quad g=\frac{p_{4}}{q_{4}} .
$$

Then we have to show that (16) implies

$$
p_{3} q_{4}=p_{4} q_{3} \quad \text { in } \quad 0 \leqslant t<t_{0} .
$$

In fact, (14) and (17) imply

(19) $\quad p_{1} q_{3}=p_{3} q_{1}$ and $\quad p_{2} q_{4}=p_{4} q_{2} \quad$ in $\quad 0 \leqslant t<T$.

Multiplying the first of equalities (19) by $q_{2}$ we obtain, in view of (16),

$$
p_{2} q_{1} q_{3}=p_{3} q_{1} q_{2} \quad \text { in } \quad 0 \leqslant t<t_{0} .
$$

Hence, by Titchmarsh's theorem on convolution,

$$
p_{2} q_{3}=p_{3} q_{2} \quad \text { in } \quad 0 \leqslant t<t_{0} \text {. }
$$

Multiplying the last equality by $q_{4}$, we obtain, in view of the second of equalities (19),

$$
p_{4} q_{2} q_{3}=p_{3} q_{2} q_{4} \quad \text { in } \quad 0 \leqslant t<t_{0},
$$

which implies (18), by Titchmarsh's theorem. 
Thus the definition of equivalency of operators $f$ and $g$ is consistent. The set $x=\{f\}$ of all operators $k$ which are equivalent to a given operator $f$ will be called the germ of the operator $f$. The concept of an operator germ is more general than that of a function germ, since every continuous function is a particular operator. Operators of the form

$$
f=\frac{p}{q}, \quad \text { where } \quad q(t)=t^{n},
$$

are distributions of finite order. The operator germs which have (20) as their representant can be called distribution germs. Distribution germs are then a particular case of operator germs. If for instance $p(t)=q(t)=t$, then $\{f\}$ is the germ of the Dirac delta distribution.

Since integrable functions are distributions, they are also operators. Thus germs of integrable functions are another particular case of operatior germs.

The set $M_{0}$ of all operator germs is a linear space.

4. An alternative definition of operator germs. Since the ring $\mathscr{C}$ has no divisors of zero, we can extend it to a quotient field $\mathscr{F}$. In other terms, elements of $\mathscr{F}$ are quotients of germs $x / y$ with $y \neq 0$. The field $\mathscr{F}$ contains $\mathscr{C}$ as its subring. Also, it contains isomorphically the field of complex numbers; we shall simply say that complex numbers are elements of $\mathscr{F}$. (They are not elements of $\mathscr{C}$, i.e. the field of complex numbers cannot be imbedded isomorphically in the ring $\mathscr{C}$; this follows from the definition of product, which is convolution in $\mathscr{C}$ ).

To every pair of functions $p, q$ from $O_{T}$ such that $q$ does not vanish identically in the neighbourhood of 0 there corresponds uniquely an element of $\mathscr{F}$ of the form $\frac{\{p\}}{\{q\}}$. Similarly, there corresponds uniquely an element of $M_{0}$ of the form $\left\{\frac{p}{q}\right\}$. Thus the pair $p, q$ determines a correspondence between elements of $\mathscr{F}$ and $M_{0}$. We shall show that this correspondence is one-to-one. To this end we have to show that

$$
\frac{\left\{p_{1}\right\}}{\left\{q_{1}\right\}}=\frac{\left\{p_{2}\right\}}{\left\{q_{2}\right\}}
$$

implies

$$
\left\{\frac{p_{1}}{q_{1}}\right\}=\left\{\frac{p_{2}}{q_{2}}\right\},
$$

and conversely. Suppose first that (21) holds. Then $\left\{p_{1}\right\}\left\{q_{2}\right\}=\left\{p_{2}\right\}\left\{q_{1}\right\}$, i.e. $\left\{p_{1} q_{2}\right\}=\left\{p_{2} q_{1}\right\}$. This means that $p_{1} q_{2}=p_{2} q_{1}$ holds in some interval $0 \leqslant t<t_{0}$. Thus we have $p_{1} / q_{1}=p_{2} / q_{2}$, provided the fractions are con- sidered as operators in $0 \leqslant t<t_{0}$. This implies (22). Suppose now, conversely, that (22) holds. Then the operators $p_{1} / q_{1}$ and $p_{2} / q_{2}$ are equal in some interval $0 \leqslant t<t_{0}$. This means that $p_{1} q_{2}=p_{2} q_{1}$ holds in $0 \leqslant t<t_{0}$. Hence we obtain successively $\left\{p_{1} q_{2}\right\}=\left\{p_{2} q_{1}\right\}, \quad\left\{p_{1}\right\}\left\{q_{2}\right\}$ $=\left\{p_{2}\right\}\left\{q_{1}\right\}$, and finally (21). Thus the correspondence between $\mathscr{F}$ and $M_{0}$ is one-to-one. It is not difficult to verify that this correspondence is an isomorphism. Thus we can identify the elements of $\mathscr{F}$ with the elements of $M_{0}$ and call them equally operator germs.

The definition given in this section has the advantage that we see at once that the set of operator germs is a field. On the other hand, the definition in section 3 is adapted better to introducing the concept of convergence of power series.

5. Power series of operator germs. As in section 2, we can consider power series (5) where $x$ is, actually, an operator germ. Imitating the previously given definition of convergence, we shall say now that series (5), where $x$ is an operator germ, converges to an operator germ $u$, if for $x=\{f\}$ (where $f$ is an operator) the corresponding power series (6) of operators converges, in some interval $0 \leqslant t<t_{0}$, to an operator $r$ such that $u=\{r\}$.

In order to make this definition sensible, we have to say what a convergence of a series of operators in an interval means, when that interval is less than the interval in which the operators are defined. Let $u_{1}, u_{2}, \ldots$ be a sequence of operators defined in an interval $0 \leqslant t<T$. We say that such a sequence converges in $I_{0}: 0 \leqslant t<t_{0}\left(0<t_{0} \leqslant T\right)$ to an operator $u$ defined in $I_{0}$, if there is a function $q$, continuous in $I_{0}$, such that all the products $q u$ and $q u_{n}$ are continuous functions in $I_{0}$ and the sequence $q u_{n}$ converges to $q u$ almost uniformly in $I_{0}$. This definition is consistent, i.e. the limit, if exists, is determined uniquely. Now, we say that a series of operators $v_{1}+v_{2}+\ldots$, defined in $0 \leqslant t<T$, converges to $v$ in a subinterval $0 \leqslant t<t_{0}$, if the sequence of partial sums $u_{n}=v_{1}+\ldots+v_{n}$ converges to $v$ in that subinterval. Thus the definition of convergence in $0 \leqslant t<t_{0}$ of a series of operators is traditional in this sense that we first define a convergence of a sequence and then the convergence of a series. On the other hand, the definition of convergence of a power series of operator germs is based directly on the convergence of series of operators, but it can be extended neither to sequences nor to arbitrary series which are not power series. Power series of operators have the same properties as expressed by formulae (10), (11) and (12).

From properties of power series of operators it is also easy to deduce other properties of power series of operator germs. For instance:

(I) If a series $\alpha_{0}+\alpha_{1} \lambda+\alpha_{2} \lambda^{2}+\ldots$ of the complex variable $\lambda$ has a positive radius of convergence, then the series $\alpha_{0}+a_{1} x+\alpha_{2} x^{2}+\ldots$ converges for every germ $x$ of an integrable function.

studia Mathematica Xxvi 7 
E.g., the series $1+x+x^{2}+\ldots$ converges for every $x$ which is a germ of an integrable function. As particularly interesting, we can consider the series

$$
1+\frac{1}{1 !} x+\frac{1}{2 !} x^{2}+\ldots
$$

This series also converges for every $x$ which is the germ of an integrable function. However, if $x$ is an arbitrary operator germ, then series (23) can be either convergent or not convergent. An operator germ $x$ for which (23) is convergent can be called, as in the Operational Calculus, a logarithm. It is natural to denote the sum of (23) by $e^{x}$. In other words, series (23) can be considered as a definition of the exponential $e^{x}$. The exponential $e^{x}$ exists for operator germs which are logarithms, and does not exist for other operator germs.

Note that in Operational Calculus the exponential is determined more generally by means of the differential equation $y^{\prime}(\lambda)=x y(\lambda)$. Such a definition cannot be used in the Calculus of Germs, for there is there no general notion of limit, and therefore no general notion of derivative.

(II) If $x$ is a logarithm, then $\lambda x$ is a logarithm, no matter what is the complex number $\lambda$ ber $\lambda$.

In other words, if $e^{x}$ exists, then $e^{\lambda x}$ exists for every complex num-

In fact, by multiplication of power series we find that if $e^{x}$ and $e^{y}$ exist for some operator germs $x$ and $y$, then also $e^{x+y}$ exists and we have

$$
e^{x+y}=e^{x} \cdot e^{y} .
$$

In particular, if $e^{x}$ exists, then $e^{2 x}=e^{x} \cdot e^{x}$. By induction we find that then also $e^{2^{n} x}$ exists for every natural number $n$. Thus (II) follows immediately from

(III) If the series

$$
\alpha_{0}+\alpha_{1} \lambda x+\alpha_{2} \lambda^{2} x^{2}+\ldots,
$$

where $\alpha_{n}$ are fixed complex numbers and $x$ is a fixed operator germ, converges for some complex number $\lambda=\lambda_{0}$, then that series converges also for every complex number $\lambda$ such that $|\lambda|<\left|\lambda_{0}\right|$.

In order to prove (III), let us represent $x$ in the form $x=\{f\}$, where $f$ is an operator. There is an interval $0 \leqslant t<t_{0}$ in which the series $a_{0}+a_{1} \lambda_{0} f+a_{2} \lambda_{0}^{2} f^{2}+\ldots$ converges operationally. By a known theorem ([3], p. 166), the series $\alpha_{0}+\alpha_{1} \lambda f+\alpha_{2} \lambda^{2} f^{2}+\ldots$ converges operationally in $0 \leqslant t<t_{0}$ for $|\lambda|<\left|\lambda_{0}\right|$. This implies that (25) converges as a power series of operator germs for every $|\lambda|<\left|\lambda_{0}\right|$.
From (24) and (III) it follows that the set of all logarithms is a linear subspace of $M_{0}$.

6. Remarks on operators. From recent results of P. Antosik and of Boehme, we can easily obtain the following theorems (IV) and (V):

(IV) If $0<T_{1}<T_{2}<\ldots$ and $T_{n} \rightarrow T$ (where $T$ may be finite or infinite) and there is a sequence of operators $x_{1}, x_{2}, \ldots$ such that $x_{n}$ is defined in $0 \leqslant t<T_{n}$ and that $x_{n}=x_{n+1}$ holds in $0 \leqslant t<T_{n}$, then there is an operator $x$ defined in $0 \leqslant t<T$ such that $x_{n}=x$ holds in $0 \leqslant t<T_{n}$ for every index $n$.

In fact, after Antosik [1], operators in open intervals $0 \leqslant t<T_{n}$ can be identified with operators in the corresponding closed intervals $0 \leqslant t \leqslant T_{n}$. I.e., each of them can be represented as a convolution quotient $x_{n}=p_{n} / q_{n}$, where $p_{n}$ and $q_{n}$ are continuous functions in $0 \leqslant t \leqslant T_{n}$. These functions can be extended continuously to the infinite interval $0 \leqslant t<\infty$. In this way also $x_{n}$ may be considered as operators in $0 \leqslant t<\infty$ with the property that $x_{n}=x_{n+1}$ holds in $0 \leqslant t \leqslant T_{n}$. After Boehme [2] there is a continuous function $q$ in $0 \leqslant t<\infty$ such that the convolution quotients $g_{n}=q / q_{n}$ are continuous functions. Evidently, we have $x_{n}=p_{n} g_{n} / q$ in $0 \leqslant t<\infty$, which implies that $p_{n} g_{n}=p_{n+1} g_{n+1}$ in $0 \leqslant t \leqslant T_{n}$. Thus $p_{n} g_{n}$ is a sequence of continuous functions which converge almost uniformly in $0 \leqslant t<T$. Its limit $p$ is a continuous function in $0 \leqslant t<T$. The operator $x=p / q$ has the required properties.

(V) Let the meaning of $T_{m}$ and $T$ be the same as in (IV). If a sequence of operators $x_{n}$ defined in $0 \leqslant t<T$ has the property that, when restricted to any of subintervals $0 \leqslant t<T_{m}$, converges in that subinterval, then it converges in the whole interval $0 \leqslant t<T$.

In fact, without loss of generality we can replace open intervals $0 \leqslant t<T_{m}$ by closed intervals $0 \leqslant t \leqslant T_{m}$. There are functions $q_{m}$, continuous in $0 \leqslant t<T$, such that the products $q_{m} x_{n}$ are continuous functions in $0 \leqslant t \leqslant T_{m}$ and that, for any fixed $m$, the sequence $q_{m} x_{n}$ converges uniformly in $0 \leqslant t \leqslant T_{m}$, as $n \rightarrow \infty$. In view of [2], there is a continuous function $q$ in $0 \leqslant t<T$ such that the convolution fractions $\frac{q}{q_{n}}$ are continuous functions in $0 \leqslant t<T$. Consequently, the sequence $q x_{n}=\frac{q}{q_{m}} \cdot q_{m} x_{n}$ consists of continuous functions and converges uniformly in each of intervals $0 \leqslant t \leqslant T_{n}$, i.e. almost uniformly in $0 \leqslant t<T$. Its limit $p$ is a continuous function in $0 \leqslant t<T$. This means that $x_{n}$ converges operationally to $\frac{p}{q}$ in $0 \leqslant t<T$.

We shall also need the following theorem: 
(VI) Let $x_{1}+x_{2}+\ldots$ be a convergent series of operators in $0 \leqslant t<T$ and $\beta_{1}, \beta_{2}, \ldots$ an absolutely convergent sequence of complex numbers, i.e. such that $\left|\beta_{2}-\beta_{1}\right|+\left|\beta_{3}-\beta_{2}\right|+\ldots<\infty$. Then the operator series $\beta_{1} x_{1}+$ $+\beta_{2} x_{2}+\ldots$ is also convergent in $0 \leqslant t<T$.

In fact, there is a function. $q$, continuous in $0 \leqslant t<T$, such that $q x_{1}+q x_{2}+\ldots$ is a sequence of continuous functions which converges almost uniformly in $0 \leqslant t<T$. We have

$$
\begin{aligned}
\beta_{m+1} q x_{m+1} & +\ldots+\beta_{n} q x_{n}=\beta_{m+1}\left(q x_{m+1}+\ldots+q x_{n}\right)+ \\
& +\left(\beta_{m+2}-\beta_{m+1}\right)\left(q x_{m+2}+\ldots+q x_{n}\right) \ldots+\left(\beta_{n}-\beta_{n-1}\right) q x_{n} .
\end{aligned}
$$

Given any subinterval $0 \leqslant t<T_{0}$ and a positive number $\varepsilon$, there exists an index $n_{0}$ such that $\left|q x_{k+1}+\ldots+q x_{n}\right|<\varepsilon$ holds in that interval for $n>k>n_{0}$. Moreover, since $\beta_{n}$ converges absolutely, there is a number $M$ such that $\left|\beta_{2}-\beta_{1}\right|+\left|\beta_{3}-\beta_{2}\right|+\ldots<M$ and $\left|\beta_{n}\right|<M$ for every $n$ Thus we have

$$
\begin{aligned}
& \left|\beta_{m+1} q x_{m+1}+\ldots+\beta_{n} q x_{n}\right| \\
& \quad \leqslant\left(\left|\beta_{m+1}\right|+\left|\beta_{m+2}-\beta_{m+1}\right|+\ldots+\left|\beta_{n}-\beta_{n-1}\right|\right) \varepsilon<2 M \varepsilon
\end{aligned}
$$

for $n>k>n_{0}$ and $0 \leqslant t<T_{0}$. Since the subinterval $0 \leqslant t<T_{0}$ is arbitrary, this proves that the series $\beta_{1} q x_{1}+\beta_{2} q x_{2}+\ldots$ converges almost uniformly in $0 \leqslant t<T$. Hence the series of operators $\beta_{1} x_{1}+\beta_{2} x_{2}+\ldots$ is convergent.

(VII) Let us consider a power series

$$
a_{0}+a_{1} f+a_{2} f^{2}+\ldots
$$

where $f$ is an operator in $0 \leqslant t<T$ and $\alpha_{n}$ are complex numbers for which the sequence $\beta_{n}=n a_{n} / a_{n-1}$ is absolutely convergent. If series (26) is convergent (operationally) in $0 \leqslant t<T$, then it remains so, if we replace $f$ by any operator $g$ in $0 \leqslant t<T$ which has the same germ as $f$.

In fact, let $T_{0}$ be any number with $0<T_{0}<T$ and let $m$ be a positive integer such that $f=g$ holds in the interval $0 \leqslant t<t_{0}=T_{0} / \mathrm{m}$. Furthermore, let $g=f+k$ in $0 \leqslant t<T$. Then $k=0$ in $0 \leqslant t \leqslant t_{0}$. This implies that $k^{n}=0$ in $0 \leqslant t \leqslant T_{0}$ for $n \geqslant m$. Consequently

$$
g^{n}=f^{n}+\left(\begin{array}{c}
n \\
1
\end{array}\right) f^{n-1} k+\ldots+\left(\begin{array}{c}
n \\
m-1
\end{array}\right) f^{n-m+1} k^{m-1} \quad \text { for } \quad 0 \leqslant t \leqslant T_{0} .
$$

\section{Hence}

$$
\begin{aligned}
& \sum_{0}^{\infty} \alpha_{n} g^{n}=\sum_{0}^{\infty} \alpha_{n} f^{n}+k \sum_{1}^{\infty}\left(\begin{array}{l}
n \\
1
\end{array}\right) \alpha_{n} f^{n-1}+\ldots+k^{m-1} \sum_{m-1}^{\infty}\left(\begin{array}{c}
n \\
m-1
\end{array}\right) \alpha_{n} f^{n-m+1} \\
= & \sum_{0}^{\infty} \alpha_{n} f^{n}+\frac{k}{1 !} \sum_{1}^{\infty} \beta_{n} \alpha_{n-1} f^{n-1}+\ldots+\frac{k^{m-1}}{(m-1) !} \sum_{m-1}^{\infty}\left(\beta_{n} \ldots \beta_{n-m+1}\right) \alpha_{n-m+1} f^{n-m+1}
\end{aligned}
$$

and after a proper change of indices

$$
\sum_{0}^{\infty} \alpha_{n} g^{n}=\sum_{0}^{\infty} \alpha_{n} f^{n}+\frac{k}{1 !} \sum_{0}^{\infty} \beta_{n+1} \alpha_{n} f^{n}+\ldots+\frac{k^{m-1}}{(m-1) !} \sum_{0}^{\infty}\left(\beta_{n+1} \ldots \beta_{n+m-1}\right) \alpha_{n} f^{n}
$$

in $0 \leqslant t \leqslant T_{0}$. Now, it is easy to show that, for every fixed $p$, the sequence $\beta_{n+1} \ldots \beta_{n+p}$ is absolutely convergent. Hence by (VI) all the series on the right-hand side of the last equality are convergent. This implies that the series on the left-hand side converges in $0 \leqslant t \leqslant T_{0}$. Since $T_{0}$ is arbitrary, the series converges in the whole interval $0 \leqslant t<T$, by (V).

Theorem (VII) applies in particular to the series

$$
1+\frac{1}{1 !} f+\frac{1}{2 !} f^{2}+\ldots
$$

In this case, the preceding argument can be made more heuristic, when we remark that the existence of the exponentials $e^{f}$ and $e^{k}$ implies the existence of the exponential $e^{g}=e^{f+k}$.

In connection with (VII) some conjectures arise. (1) Does theorem (VII) remain true if the assumption on the coefficients $a_{n}$ is released ? (2) Can we assert that the convergence of (27) in a subinterval $0 \leqslant t<t_{0}$ implies the convergence of $(27)$ in the whole interval $0 \leqslant t<T$ ? Or, in other words, is the convergence of (27) a consequence of the convergence of the corresponding sequence of germs?

\section{References}

[1] П. Антосик, Об изоморфивме некоторых колец операторов, Studia Math. 25 (1965), p. $227-230$.

2] $\mathrm{T}$, B on sequences of continuous functions and convolution, (1965), p. 333-335.

[3] J. Mikusiński, Operational Calculus, Warszawa 1959. $225-251$. 